\title{
Nonlinear optical properties of ytterbium-doped tantalum pentoxide rib waveguides on silicon at telecom wavelengths
}

\author{
C. Lacava ${ }^{(1), *}$, A. Aghajani ${ }^{(1)}$, P. Hua ${ }^{(1)}$, D.J. Richardson ${ }^{(1)}$, P. Petropoulos ${ }^{(1)}$, J. S. Wilkinson ${ }^{(1)}$ \\ (1) Optoelectronics Research Centre, University of Southampton, Highfield Campus, SO17 1BJ, Southampton, United Kingdom \\ *C.Lacava@soton.ac.uk
}

\begin{abstract}
We report the linear and nonlinear characterization of low loss $\mathrm{Yb}: \mathrm{Ta}_{2} \mathrm{O}_{5}$ rib waveguides fabricated by using CMOS compatible processes on a SOI substrate. We report a nonlinear refractive index of $6.65 \times 10^{-18} \mathrm{~m}^{2} / \mathrm{W}$ measured at telecom wavelengths.

OCIS codes: (190.4400) Nonlinear optics, materials; (190.4380) Nonlinear optics, Four Wave Mixing; (190.4390) Nonlinear optics, integrated optics.
\end{abstract}

\section{Introduction}

Next generation high-capacity communications will require extremely high-speed and low-power optical components for all-optical signal processing. In recent years, the integration of several optical functionalities on the same chip has attracted the attention of the scientific community and considerable advances have already been achieved in the last decade. The inclusion of nonlinear optical components in integrated devices is often highly desirable, because of their capability to efficiently manipulate and process optical signals. The implementation of several nonlinear processing functions, such as all-optical wavelength conversion [1], regeneration of phase-encoded signals [2], Wavelength Division Multiplexing (WDM) routing [1] and all-optical modulation [3], has already been demonstrated in integrated devices. Most of the attention is currently focused on the Silicon Photonics integration platform that already provides excellent and reliable integrated components [1]. Silicon on Insulator (SOI) technology serves as the fabrication platform for silicon photonics components and, thanks to the extremely high refractive index contrast $(>2)$, offers the possibility to realize waveguides with very tightly confined optical modes. By also considering the large refractive index of silicon at $1550 \mathrm{~nm}$, SOI waveguides represent the ideal candidate for realizing low power, integrated nonlinear optical components [1]. Unfortunately, silicon also exhibits an extremely high Two-Photon Absorption (TPA) coefficient at the same wavelength range, preventing the realization of practical components and limiting the overall nonlinear efficiency of the final device.

In recent years, different solutions have been proposed to overcome this limitation and, at the same time, preserve the undeniable advantage offered by SOI technology. Silicon nitride $\left(\mathrm{Si}_{3} \mathrm{~N}_{4}\right)$ has recently emerged as a viable alternative [4] but a careful control of the induced stress on the $\mathrm{SiO}_{2}$ substrate is required, making the development of relatively thick waveguides $(>300 \mathrm{~nm})$ difficult [4]. Moreover the nonlinear refractive index of $\mathrm{Si}_{3} \mathrm{~N}_{4}$ is almost one order of magnitude lower than that observed in Silicon, requiring relatively long waveguide sections (order of meters) to achieve the desired effect [4]. Amorphous Silicon has also shown potential but many groups have reported material instability [5] and further research efforts have still to be committed. AlGaAs also shows promising characteristics [6] and has been recently successfully integrated on a SOI platform [6] leading to a very good performance waveguide. However, this approach still requires a wafer bonding technique to be applied, which is not desirable for passive waveguide components and adds several steps to the fabrication process relative to the standard SOI procedure. Tantalum pentoxide $\left(\mathrm{Ta}_{2} \mathrm{O}_{5}\right)$ on the other hand, is a CMOS compatible material that has been studied in the past for its nonlinear applications at $980 \mathrm{~nm}$ and has only very recently been considered as a possible alternative for telecom wavelength applications [7]. Thanks to the extremely large band-gap value (3.8 eV), no TPA effect is present at the $1550 \mathrm{~nm}$ wavelength region, making this material an ideal candidate for nonlinear optical components integrated on a SOI substrate. Here, we study for the first time at wavelengths relevant to telecommunications, the nonlinear optical properties of low loss $\mathrm{Yb}: \mathrm{Ta}_{2} \mathrm{O}_{5}$ rib waveguides integrated on the top of a $\mathrm{SiO}_{2}$ layer. Through continuous wave (CW) Four-Wave Mixing (FWM) experiments, we have evaluated the nonlinear parameter $\gamma$ and by using the simulated effective area we have extracted the nonlinear refractive index to be equal to $6.65 \times 10^{-18} \mathrm{~m}^{2} / \mathrm{W}$, which is of the same order of magnitude as in silicon. We have also measured the linear propagation losses of the waveguide to be $0.19 \mathrm{~dB} / \mathrm{cm}$. It is worth noting that $\mathrm{Ta}_{2} \mathrm{O}_{5}$ has also been investigated as a gain material for laser applications $[8,9]$ and it has been proven that it can be used as a host material for many dopants adding optical functionality, therefore the integration of $\mathrm{Ta}_{2} \mathrm{O}_{5}$ on the silicon platform could introduce several functionalities that are vital for the development of a full operational, multifunctional, optical transceiver for next generation optical communications. 


\section{Device fabrication}

A $1 \mu \mathrm{m}$ thick $\mathrm{Yb}: \mathrm{Ta}_{2} \mathrm{O}_{5}$ layer was deposited by RF magnetron sputtering from a powder-pressed $\mathrm{Ta}_{2} \mathrm{O}_{5}$ target doped with $2.5 \mathrm{wt} . \%$ of $\mathrm{Yb}$ oxide $\left(6.2 \times 10^{20} \mathrm{Yb}\right.$ ions $\left./ \mathrm{cm}^{3}\right)$ [8] on to a silicon substrate with a $2.5 \mu \mathrm{m}$ thermally-grown silica layer. The following conditions were used for the deposition process: 20 and $5 \mathrm{sccm}$ of argon and oxygen gas flow, respectively, substrate temperature of $200{ }^{\circ} \mathrm{C}$ and a magnetron power of $300 \mathrm{~W}$; the chamber was set at a pressure of 10 mTorr. Shallow rib waveguide channels with a width between $1 \mu \mathrm{m}$ and $10 \mu \mathrm{m}$ were defined in the $\mathrm{Yb} \mathrm{Ta}_{2} \mathrm{O}_{5}$ layer using standard photolithography and etched by $150 \mathrm{~nm}$ using argon ion beam milling. The rib was encapsulated with a $1.6 \mu \mathrm{m}$ thick $\mathrm{SiO}_{2}$ over-cladding, deposited using $\mathrm{RF}$ magnetron sputtering, creating a symmetrical waveguide. The wafer was then diced and the end-facets were optically polished for characterization.

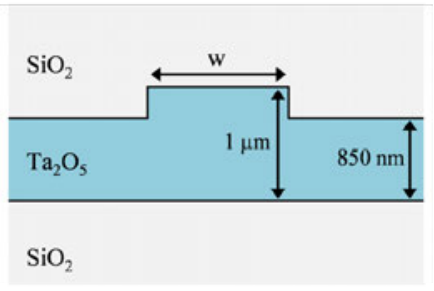

(a)

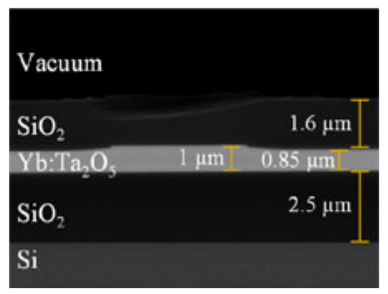

(b)

Fig.1: (a) Cross section of the $\mathrm{Ta}_{2} \mathrm{O}_{5}$ RIB waveguide, (b) SEM image of the fabricated final device covered by $1.6 \mu \mathrm{m}$ of $\mathrm{SiO} \mathrm{O}_{2}($ width $=5 \mu \mathrm{m})$

Fig. 1(a) shows the cross section of the target device. The refractive index of $\mathrm{Yb}: \mathrm{Ta}_{2} \mathrm{O}_{5}$ at $1550 \mathrm{~nm}$ is 2.115 which gives a refractive index contrast of $\sim 1.43$. A Scanning Electronic Microscope (SEM) image is shown in Fig. 1(b). The width $w$ of the waveguide shown in this image is equal to $5 \mu \mathrm{m}$.

\section{Linear and nonlinear optical characterization}

In order to assess the nonlinear performance of our waveguides we only considered the $1 \mu \mathrm{m}$-width device that exhibits the highest nonlinearities. As a first step, we assessed the linear propagation losses by using a cut-back scheme. A CW laser was used as a source and the optical beam was injected in the waveguide under test by means of a tapered optical fiber with the aim of reducing the coupling losses. A 99:1 tap fiber coupler was placed before the launch fiber in order to carefully measure the power level at the input. A polarization controller was also used to set the state of polarization of the light. After propagation in the waveguide, the optical beam was collected using an identical tapered optical fiber and the light was then sent to a power meter to measure the output optical power. By measuring different length waveguides (in the range of $1 \mathrm{~cm}$ to $3 \mathrm{~cm}$ ) we were able to extract both the coupling loss and the propagation loss values which were equal to $6.33 \mathrm{~dB} /$ facet and $0.19 \mathrm{~dB} / \mathrm{cm}$, respectively.

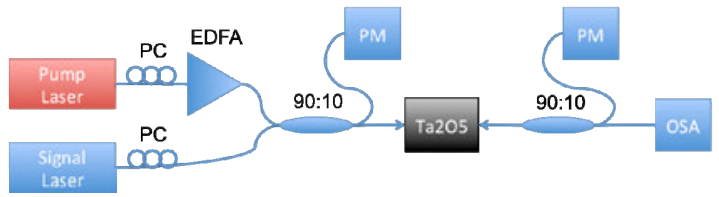

Fig.2: Four Wave Mixing experimental set-up. PC: Polarization Controller, PM: Power Meter, OSA: Optical Spectrum Analyzer.

As a second step, we assessed the nonlinear performance of our device at $1550 \mathrm{~nm}$. In order to measure the nonlinear parameter $\operatorname{Re}\{\gamma\}$ we used the experimental set-up shown in Fig.2. Light from a pump laser was amplified using a high-power EDFA and coupled together with a weaker signal by means of a 90:10 fiber coupler. Two polarization controllers were used in each branch to set the polarization of the optical beams. A pair of tapered fibers was used to inject and collect back the light from the waveguide under test. The beam was then sent to an optical spectrum analyzer (OSA) in order to observe the optical spectra at different power levels and measure the FWM efficiency. The power levels at the input and output of the waveguide were carefully monitored through tap couplers. The relationship between $\operatorname{Re}\{\gamma\}$, the FWM efficiency, the waveguide length and the input optical power is expressed by the following relation: $P_{i}(L) / P_{s}(L)=\left(\operatorname{Re}\{\gamma\} L_{e f f}\right)^{2} P_{p}(0)^{2}$, where $P_{i}(L)$ and $P_{s}(L)$ are the idler and signal power levels at the output of the waveguide respectively, $L_{\text {eff }}$ represents the nonlinear waveguide length and $P_{p}(0)$ the pump power at the input of the waveguide [10]. Therefore, by measuring the FWM-strength $\left(P_{i}(L) / P_{s}(L)\right)$ for different pump power levels, and by knowing the waveguide length, it is possible to extract the nonlinear parameter $\operatorname{Re}\{\gamma\}$. 


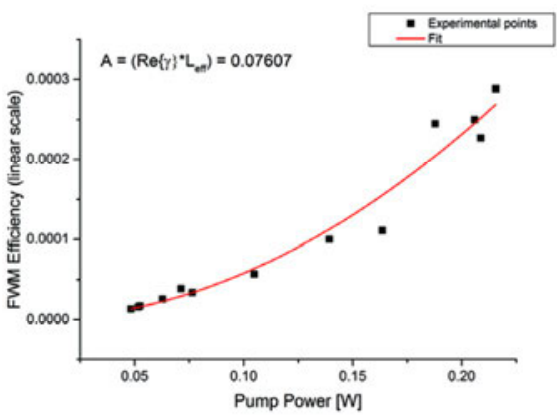

(a)

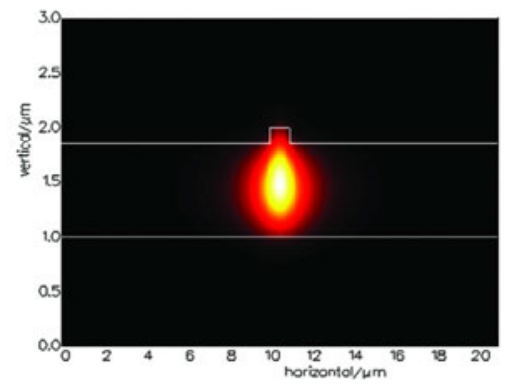

(b)

Fig.3: (a) Measured FWM efficiency as a function of the pump power for $L=1 \mathrm{~cm}$ waveguide. The fit parameter "A" represents Re $\{\gamma\}\}^{*} L_{e f f}$ (b) The simulated optical mode profile (waveguide width $=1 \mu \mathrm{m}$ ). Please note that the $\mathrm{x}$ and $\mathrm{y}$ axes are on different scales.

Figure 3 shows the experimental results together with the fitting function for a waveguide length of $1 \mathrm{~cm}$. A maximum efficiency of $-37 \mathrm{~dB}$ has been demonstrated for the first time, to the best of our knowledge, in a $\mathrm{Yb}: \mathrm{Ta}_{2} \mathrm{O}_{5}$ waveguide at $1550 \mathrm{~nm}$. The fitting parameter $\mathrm{A}=\operatorname{Re}\{\gamma\}^{*} L_{\text {eff }}$ (as shown in Fig.3) has been assessed and is equal to 0.0760 . Therefore, considering a waveguide length of $1 \mathrm{~cm}, \operatorname{Re}\{\gamma\}=7.81(\mathrm{Wm})^{-1}$. In order to extract the nonlinear refractive index $\mathrm{n}_{2}$, we simulated the optical mode distribution of our waveguide (width=1 $\mu \mathrm{m}$ ) by using the commercial software PhotonD ${ }^{\odot}$. An effective area $A_{\text {eff }}$ of $3.47 \mu \mathrm{m}^{2}$ was calculated, which yielded a value of $6.65 \times 10^{-18} \mathrm{~m}^{2} / \mathrm{W}$ for the nonlinear refractive index at $1550 \mathrm{~nm}$. This value is comparable to that of silicon $\left(2 \times 10^{-18} \mathrm{~m}^{2} / \mathrm{W}\right)$.

\section{Conclusions}

In this work we have demonstrated low loss $(0.19 \mathrm{~dB} / \mathrm{cm})$ highly nonlinear $\mathrm{Yb}: \mathrm{Ta}_{2} \mathrm{O}_{5}$ rib waveguides, fabricated with CMOS compatible processes on a SOI substrate, for all-optical signal processing at telecom wavelengths. We have measured a nonlinear refractive index of $\mathrm{n}_{2}=6.65 \times 10^{-18} \mathrm{~m}^{2} / \mathrm{W}$ which is promising for nonlinear applications. Thanks to its nonlinear properties, $\mathrm{Ta}_{2} \mathrm{O}_{5}$ could form a suitable material for the implementation of nonlinear applications on a SOI substrate, overcoming some of the drawbacks of Si associated with the onset of nonlinear losses.

\section{Acknowledgments}

This work has been partially supported by EPSRC, UK through the SPFS Programme Grant and the Integrated Photonic Materials and Devices Platform Grant. http://dx.doi.org/10.5258/SOTON/382841

\section{References}

[1] B. Jalali and F. Sasan, "Silicon photonics," J. Light. Technol., vol. 24, no. 12, pp. 4600-4615, Dec. 2004.

[2] F. Da Ros, D. Vukovic, A. Gajda, K. Dalgaard, L. Zimmermann, B. Tillack, M. Galili, K. Petermann, and C. Peucheret, "Phase regeneration of DPSK signals in a silicon waveguide with reverse-biased p-i-n junction.," Opt. Express, vol. 22, no. 5, pp. 5029-36, 2014.

[3] C. Lacava, M. J. Strain, P. Minzioni, I. Cristiani, and M. Sorel, "Integrated nonlinear Mach Zehnder for 40 Gbit/s all-optical switching," Opt. Express, vol. 21, no. 18, p. 21587, Sep. 2013

[4] D. J. Moss, R. Morandotti, a L. Gaeta, and M. Lipson, "New CMOS-compatible platforms based on silicon nitride and Hydex for nonlinear optics," Nat. Photonics, vol. 7, no. July, pp. 597-607, 2013.

[5] B. Kuyken, H. Ji, S. Clemmen, S. K. Selvaraja, H. Hu, M. Pu, M. Galili, P. Jeppesen, G. Morthier, S. Massar, L. K. Oxenløwe, G. Roelkens, and R. Baets, "Nonlinear properties of and nonlinear processing in hydrogenated amorphous silicon waveguides," Opt. Express, vol. 19, no. 26, p. B146, 2011.

[6] M. Pu, H. Hu, L. Ottaviano, E. Semenova, D. Vukovic, L. K. Oxenlowe, and K. Yvind, "AlGaAs-On-Insulator Nanowire with 750 nm FWM Bandwidth, -9 dB CW Conversion Efficiency, and Ultrafast Operation Enabling Record Tbaud Wavelength Conversion," in Optical Fiber Communication Conference Post Deadline Papers, 2015, p. Th5A.3.

[7] C. Wu, Y. Chiu, C. Chen, Y. Lin, A. Chu, and C. Lee, "Four-wave-mixing in the loss low submicrometer Ta2O5 channel waveguide," Opt. Lett. 40, 4528-4531 (2015)

[8] A. Aghajani, G. Murugan, N. Sessions, S. Pearce, V. Apostolopoulos, and J. Wilkinson, "Spectroscopy of ytterbium-doped tantalum pentoxide rib waveguides on silicon," Opt. Mater. Express 4, 1505-1514 (2014).

[9] Subramanian, A.Z.; Oton, C.J.; Shepherd, David P.; Wilkinson, J.S., "Erbium-Doped Waveguide Laser in Tantalum Pentoxide," in Photonics Technology Letters, IEEE, vol.22, no.21, pp.1571-1573, Nov.1, 2010

[10] Agrawal, Govind P. Nonlinear fiber optics. Academic press, 2007. 